\title{
Comparing the effect of pool therapy and land therapy on balance in elderly population
}

\author{
K. Vadivelan ${ }^{1 *}$ and Shravanthi ${ }^{1}$ \\ ${ }^{1}$ SRM College of Physiotherapy, SRM Institute of Science and Technology, Kattankulathur, \\ Kancheepuram District, Chennai 603203, India. \\ *Corresponding E-mail: vadivelk@,srmist.edu.in
}

\begin{abstract}
Balance is defined as the dynamic process by which the position of the body is maintained in equilibrium. As age increases balance decreases that lead to falls. To improve the balance, land, and pool therapies are used. Studies have shown that land-based exercises improve joint flexibility, muscle strength, balance, and reaction time in an older population. The physical properties of water are an effective rehabilitation program. Water-based exercises provide a viable alternative for the elderly who are unable to participate in land-based exercise programs as water exercise has reduced the risk of falls. The objective of the study is to compare the effect of pool therapy and land therapy on balance in the elderly population. The Study methodology: Quasi-experimental study.30 elderly people between 60-75 years (both male and female) were included in the study based on inclusion and exclusion criteria. Tinetti falls efficacy scale questionnaire was given to the subjects and they were asked to fill it and then a timed up and go test was done before and after the exercise protocol. The 30 individuals were divided into 2 groups of 15 members each (Group A and Group B). Group A was given pool therapy and Group B was given land-based therapy for 6 weeks. The results of statistical analysis showed a significant difference between land therapy and pool therapy. This study concludes that pool therapy is more effective in improving balance in the elderly population.
\end{abstract}

Keywords: pool therapy, balance, land therapy, fall, elderly population.

Received: 11 October, 2021

Accepted: 24 October, 2021

Published: 31 October, 2021

\section{Introduction}

The global population of people above the age of 60 years is $12.3 \%$ and India is the second-highest populated country in the world having 766 lakhs people above the age of 59, compromising above $6.24 \%$ of the entire population. The main concern of the old age population is their health which is mainly affected by various medical and psychological issues. Falls among the elderly population is considered as "Geriatric Giants" as it is one of the major problems [19]. According to WHO fall is defined as an event that results in a person coming to rest inadvertently on the ground or floor or other lower level. Fall-related injuries may be fatal or non-fatal though most are non-fatal [18]. Frequent falling is a vital cause for morbidity and mortality in the elderly population and it indicates deprived physical and cognitive status [13, 15]. Fall has many etiology like muscle weakness, arthritis, use of more than three prescribed medications, depressions, use of an assistive device, impairments in gait, balance, vision, cognition, and activities of daily living [28]. 
The incidence of falls among Indian older adults ranges from $14 \%$ to $53 \%$ with fractures up to $21.3 \%$ and other injuries up to $79.6 \%$ and the burden of falls is $26 \%$. Fractures are more common in females (26.4\%) than males $(16 \%)$ and the fracture percentage is more in the urban population $(29.4 \%)$ than rural population $(13.4 \%) 2,10$ The percentage of old age population which is above 59 years has raised from $9 \%$ in 1994 to $12 \%$ in 2014 and it is expected to reach $21 \%$ in 2050 [21]. A country is labeled as "aging" when $7 \%$ of the population is aged 60 years and above. India exceeded this mark in 2000 and by 2025 it is expected to reach $12.6 \%$. This highlights the fact that India is moving from a young nation to a graying nation [18]. Tamil Nadu has $11.2 \%$ of the elderly population and is ranked behind Kerala which has $12.3 \%$. Most falls are predictable and can therefore be avoided Most the falls in the elderly population result in a hip fracture that can be prevented by constant exercise that improves muscle strength, coordination and flexibility, changes in the environment, and other educational opportunities.1 However, further studies are required to investigate the effectiveness of these strategies in the Indian elderly population. Regular exercise and balance training programs can diminish the danger of falling by $10 \%$ and $20 \%$ respectively [14]. The morbidity that occurs with falls includes visual and vestibular impairment, cognition, syncope, neurological and musculoskeletal disorders, medication, severe injuries and fractures, limited mobility and dependency leading to functional decline, phobia of falling (post-fall syndrome), and perpetual disability. Therefore fall is a public health issue that should be prevented in order to prevent various other conditions [24].

Hence this study has been taken in order to compare two techniques (Pool Therapy and Land-Based Therapy) for improving the balance in the elderly population and thereby preventing falls. Pool Therapy uses water for the rehabilitation of patients. The properties of water along with exercise are used for effective rehabilitation. In an aquatic environment, the joint load and the risk of falls and lesions are reduced [28]. The water environment is considered safe and effective for the old age population due to buoyancy. Most falls are predictable and can therefore be avoided. Balance is defined as a dynamic process by which the position of the body is maintained in equilibrium [9]. There are many factors that are responsible for the loss of balance in the elderly population [12]. Balance is reduced in the elderly population due to deterioration of the postural control system and compromised central nervous system mainly and that is caused due to aging [3]. Postural control is the ability of the body to maintain postures such as standing, moving independently, and preventing falls when the balance is lost. As a person ages, the system that controls the posture deteriorates which causes balance deficiencies, incompetence to react properly, limitation of mobility thus ultimately leading to disability [25]. Central nervous system helps in vestibular, proprioceptive, and visual signals that maintain body balance and individual ability to adaptive reactions. The consequences of falls are acute injuries, traumatic injuries, spinal cord injuries, brain injuries, hip fractures, and sometimes even death. Hence injuries associated with falls are a serious public health problem [22]. There are various studies that show the effect of land-based exercises on balance in older people but there are limited reports on the effects of water therapy on balance [11]. Hence this study has been articulated to compare the effect of pool therapy and land-based therapy on balance in the elderly population.

\section{Aim of the study}

To compare the effect of pool therapy and land therapy on balance in an elderly population.

\section{Methodology}

The study design is a quasi-experimental study of comparative type done for 6 weeks. 30 samples were selected through convenient sampling in private apartments with pools in and around Chennai.

\section{Procedure}


30 samples were taken between the age group of 60-75 years who are able to walk and carry out their activities of daily life independently and who do not have any dermatological conditions, urinary and fecal incontinence, dyspnea, cancer and use of psychotropic drugs. The assessment started with signing the consent form and an interview to collect rudimentary information such as demographic data, past history, and present history which included the number of falls in last year. After the interview, their balance was evaluated using TUG test and they were given Tinetti FES to fill. The TUG test is used in detecting the balance related issues that affect the elderly population activities of daily life. The test should be completed in a short duration. In this test, participants were seated in a chair $3 \mathrm{~m}$ from a line. Once the timer instructed the participant to "go," they rose from the chair (without using their hands), walked to the line, and returned to sit back down. The aim of this test was to complete it as fast as possible, as the total time is recorded on a stopwatch from when the participant was instructed to "go" and is stopped when they sat back down. Then the participants are divided into 2 groups:

Group 1: Participants were given pool therapy.

Group 2: Participants were given land-based exercises.

The Tinetti FES and TUG test was applied before the exercise protocol (pre-test) and after 6 weeks of pool therapy or land-based exercises (post-test). This was done to evaluate the effect of the exercises and to find which protocol is better.

This study includes balance exercises for elders that were given for six weeks. These exercises were done twice a week which has one set of exercises that were repeated two times.

The land-based therapy includes side way walking, simple grapevine, marching on one place, walking and turning around, stepping over objects, tandem walking, step up, and one leg stance. These were done in progression where the first four exercises were done for 2 weeks after which the stepping over objects and tandem walking was added to the protocol which was practiced for the third week and all the 8 exercises were done for the fourth week and in fifth and sixth week all the 8 exercises were done three times a week.

The pool therapy included standing with support (wall of the pool) for the first week so that they get oriented with the water environment. For the second week they were made to stand without any support and third week they stood on one leg with support and without support on the fourth week. In the fifth-week sideway walking, marching on the spot and walking and turning around was done with 2 repetitions and for the sixth week, they were made to repeat the exercises for 3 times.

\section{Data Collection}

30 samples were taken according to the inclusion and exclusion criteria and with the history of at least 2 falls in the previous year from 3 private apartments which have pools with security.

\section{Results}

Table I shows a comparison of pre-test and post-test results of TUG test and Tinetti FES of land therapy among the elderly population. Land therapy has a p-value of 0.00 in Tinetti FES which is significant and in TUG test with 0.07 which is not significant. The mean difference of TUG test in land therapy is 0.63 . The Tinetti FES has a mean difference of 5.67 in land therapy.

Table I: Comparison between pre-test and post-test of group B (land therapy) 


\begin{tabular}{|c|l|c|c|}
\hline & & Mean & Mean Difference \\
\hline \multirow{2}{*}{$\begin{array}{c}\text { Land } \\
\text { Therapy }\end{array}$} & Pre-Test TUG & 12.28 & \multirow{2}{*}{0.63} \\
\cline { 2 - 3 } & Post-Test TUG & 11.65 & \\
\hline \multirow{2}{\text{Land}}{\begin{tabular}{c} 
Therapy \\
\cline { 2 - 3 }
\end{tabular}} & Pre-Test FES & 69.4 & \multirow{2}{*}{5.67} \\
\cline { 2 - 3 } & Post-Test FES & 63.73 & \\
\hline
\end{tabular}

$\mathrm{P}<0.005$ shows significant results

Table I shows the comparison of pre-test and post-test values of Group B. The mean value of 12.28 in pre-test and 11.65 in post-test of TUG in Group B

Table II shows the comparison between pre-test and post-test results of pool therapy in TUG test and Tinetti FES. A p-value of 0.002 in Tinetti FES and 0.00 in Timed up and go test was found which are significant. The mean difference of 0.63 is seen in TUG test and 6.07 is seen in Tinetti FES in pool therapy.

Table II: Comparison between pre-test and post-test of group A (pool therapy)

\begin{tabular}{|c|l|c|c|}
\hline & & Mean & Mean Difference \\
\hline \multirow{2}{*}{$\begin{array}{c}\text { Pool } \\
\text { Therapy }\end{array}$} & Pre-Test TUG & 11.51 & \multirow{2}{*}{0.63} \\
\cline { 2 - 3 } $\begin{array}{c}\text { Pool } \\
\text { Therapy }\end{array}$ & Post-Test TUG & 10.88 & \\
\cline { 2 - 3 } & Pre-Test FES & 79.07 & \multirow{2}{*}{6.07} \\
\hline
\end{tabular}

$\mathrm{P}<0.005$ shows significant results

The mean value of post-test of FES in group A is 73 while it is 63.73 in group B. The mean value of TUG test in group $\mathrm{A}$ is 10.88 and group $\mathrm{B}$ is 11.65

Table III shows the comparison between post-test results of pool therapy and land therapy in TUG test and Tinetti FES. The p-value of the post-test results of land therapy and pool therapy in TUG test and Tinetti FES is 0.00 which is significant. The mean value of post-test of TUG test in pool therapy is 10.88 and in land therapy is 11.65 . The mean value of 63.73 and 73 was found in the post-test results of Tinetti FES of land therapy and pool therapy respectively.

Table III: Comparison between post- tests of group A and group B

\begin{tabular}{|c|l|c|c|}
\hline & & Mean & Std. Deviation \\
\hline $\begin{array}{c}\text { Post-test of } \\
\text { FES }\end{array}$ & Land therapy & 63.73 & 12.37 \\
\cline { 2 - 4 } & Pool therapy & 73 & 8.65 \\
\hline $\begin{array}{c}\text { Post-test of } \\
\text { TUG }\end{array}$ & Land therapy & 11.65 & 1.28 \\
\cline { 2 - 4 } & Pool therapy & 10.88 & 1.05 \\
\hline
\end{tabular}

$\mathrm{P}<0.005$ shows significant results

The mean value of 69.4 in pre-test and 63.73 in post-test of FES in group A was found.

The bar graph I depict the comparison of pre-tests and post-tests of TUG test and Tinetti FES land therapy. The 
mean value of pre-test of TUG test for land therapy is 12.28 and post-test is 11.65 with a mean difference of 0.633 . The mean value of pre-test and post- test of Tinetti FES for land therapy is 69.4 and 63.73 respectively with a mean difference of 5.67 .

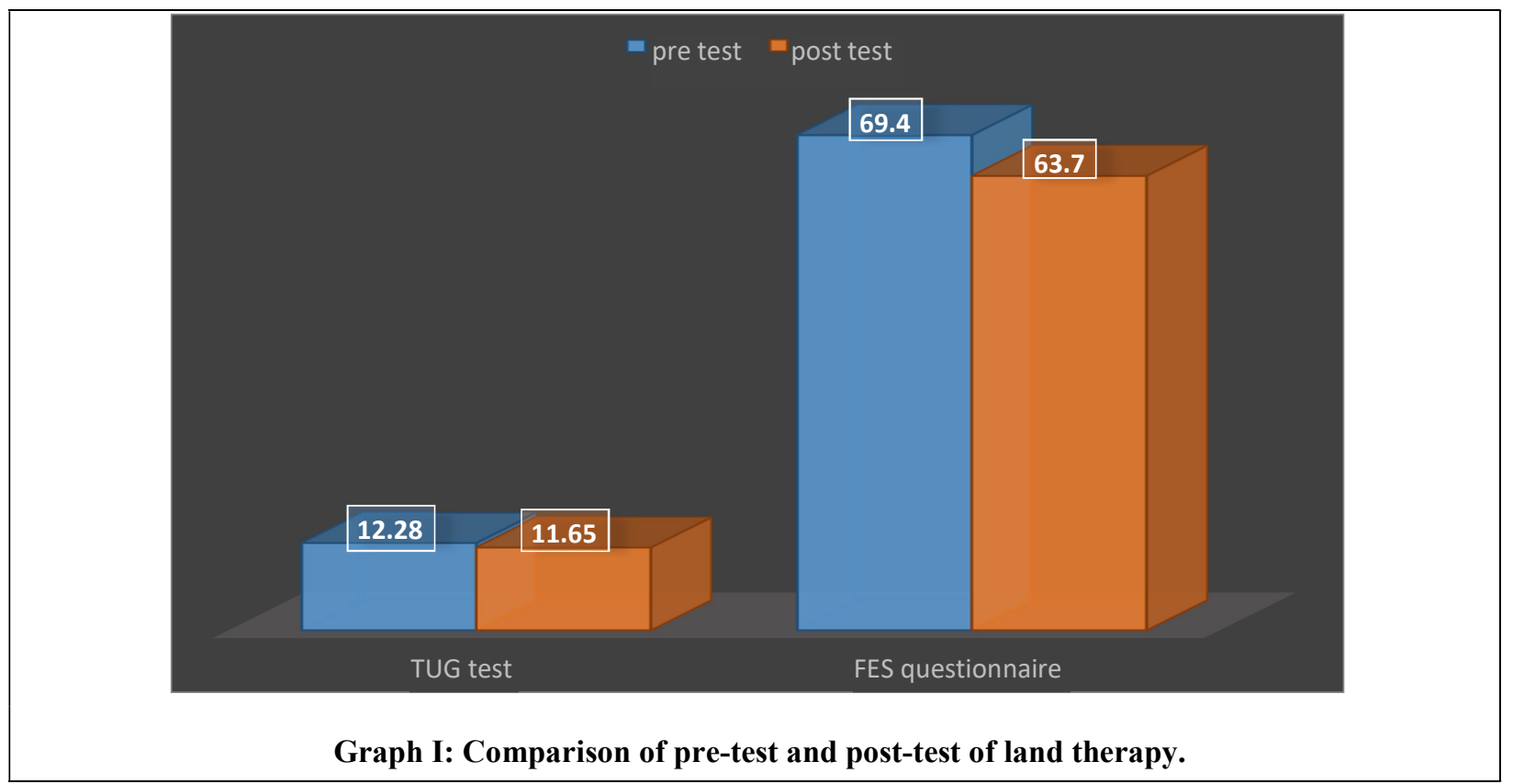

The bar graph II shows the comparison of pre-test and post-test of TUG test and Tinetti FES of pool therapy. The mean value of pre-test and post-test of TUG test for pool therapy is 11.51 and 10.88 respectively with a difference of 0.63 . The mean value of pre- test and post-test of Tinetti FES for pool therapy is 79.07 and 73 respectively and the mean difference is 6.07. The bar graph III shows the comparison between post-test results of TUG test and Tinetti FES of both pool therapy and land therapy.

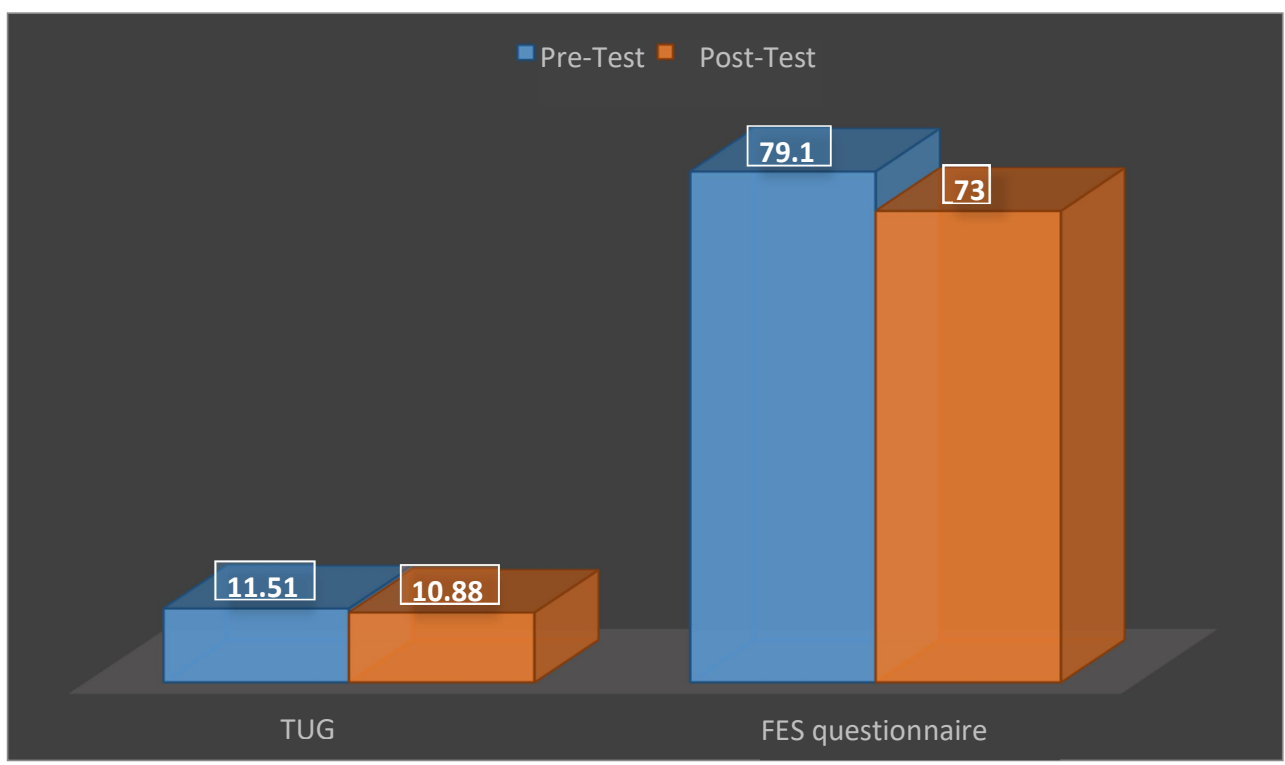

Graph II: Comparison of pre-test and post-test of land therapy. 


\section{Discussion}

The objective of this study was to explore whether the balance exercises, conducted either in the pool or on the land environment, would be capable of improving the balance in the elderly population. In order to test the hypothesis, exercises were used that are capable of being reproduced and applied easily. This study is mainly concerned with balance exercises for the elderly population as they are the high-risk group for falls. Studies reveal that there is an increasing rate of falls in the elderly population recently. They fall frequently due to changes related to aging. Aging comprises the capability of the central nervous system to process the vestibular, visual, and proprioceptive signals that are accountable to retain body balance as well as reducing the individual's capability to cope up with adaptive reactions limiting functional mobility which is responsible for making the older adults more dependent [29]. Fall has many etiology like muscle weakness, arthritis, a history of falls, use of four or more prescription medications, depression, and use of an assistive device, impairments in gait, balance, vision, cognition, and activities of daily living [28].

There are studies that have used land-based exercises to improve balance and there are studies that use water to increase balance in the elderly population separately. But there are only a few studies that compare both the therapies to investigate which has better results. Land-based exercises can be done at home with minimum support at any time. Pool therapy demands less from the body and it also reduces the joint load [5]. Buoyancy and water pressure can be utilized as resistance for strengthening exercises [6]

This study includes balance exercises for elders that were given for six weeks. These exercises were done twice a week which has one set of exercises that were repeated two times. There is an increase in balance in pool therapy as it is a proprioceptive training that increases the stability by providing time to stimulate the postural reaction to perturbations in the environment [27] and also due to the properties of water such as density, hydrostatic pressure, viscosity, buoyancy, specific gravity, and thermodynamics. Immersion of the body improves balance abilities by increasing proprioception, body alignment, stability, and sensory feedback. As resistance to movement in water is greater than air the sensory output to the muscles increases and the muscles contract to stabilize the postural alignment $[16,26]$. The improvement in land therapy is produced by the change in the anticipatory center of pressure and the feedback to maintain balance and also by the development in the somatosensory system which includes proprioceptive and tactile sensors [20,30]. The exercises helped in improving the body righting and equilibrium through higher cortical reactions facilitation (protective, equilibrium, and righting reactions) [7,8].

According to the statistics group B have a significant value in TUG test but it doesn't have a significant value in FES whereas both TUG and FES have a significant value in group A. Hence pool therapy is effective in improving balance among the elderly population. This result is contradicted by Nubia C.P. Avelar et.al., Robyn Lin Anderson who found that there is no significant difference between the land and aquatic group [17, 29].

Lund. H et.al., (2010) and Marco Bergamin (2013) compared the aquatic and land-based exercises and found that aquatic exercise has a better result and these studies support this study [4, 23]. Hence a conclusion can be drawn that pool therapy can be used to improve balance effectively in the elderly population.

\section{Limitations and Recommendations}

\section{Limitations}

This study was carried out for a short duration of 6 weeks with only 2 outcome measures. As convenient sampling was done samples were not segregated into land therapy and pool therapy through the proper method. The quality of life was not analyzed. 


\section{Recommendations}

Duration of study can be elongated with more outcome measures and proper sampling methods along with the longterm benefits of the rehabilitation protocol.

\section{Conclusion:}

Both land therapy and pool therapy have a significant value in the TUG test wherein FES pool therapy alone has a significant value. Hence a conclusion can be drawn that when comparing the effects of pool therapy and land therapy on balance in elderly population, pool therapy is effective in improving balance among the elderly population.

\section{DECLARATIONS}

\section{Funding}

This research did not receive any specific grant from funding agencies in the public, commercial, or not-for-profit sectors.

\section{Conflicts of Interest}

The authors declared no potential conflicts of interest with respect to the research, authorship, and/or publication of this article.

\section{Acknowledgment}

First and foremost, I would like to thank my parents and my guide Mr. K.Vadivelan, M.P.T., Associate Professor for their encouragement and support at all levels. Last but not least, I would like to thank my sister and my close ones for their valuable suggestions and support in the completion of my project.

\section{Availability of data and materials}

The datasets used and/or analyzed during the current study are available from the corresponding author on reasonable request. Please write to vadivelk@srmist.edu.in, karulvela@gmail.com

\section{REFERENCES}

[1] Chan K, Phadke CP, Stremler D, Suter L, Pauley T, Ismail F, Boulias C. The effect of water-based exercises on balance in persons post-stroke: a randomized controlled trial. Topics in stroke rehabilitation. 2017 May 19;24 (4):22835.

[2] Van Voast Moncada la. Management of falls in older persons: a prescription for prevention. American family physician. 2011 Dec 1;84 (11).

[3] Guo LY, Yang CP, Yu YL, Chen SK, Yang CH, Hou YY, Wu WL. Underlying mechanisms of Tai-Chi-Chuan training for improving balance ability in the elders. Chinese journal of integrative medicine. 2014 Jun 1;20(6):409-15.

[4] Lund H, Weile U, Christensen R, Rostock B, Downey A, Bartels EM, Danneskiold- Samsøe B, Bliddal H. A randomized controlled trial of aquatic and land-based exercise in patients with knee osteoarthritis. Journal of rehabilitation medicine. 2008 Feb 5;40(2):137.

[5] Yoo JS, Jeon MY, Kim CG. Effects of a Fall Prevention Program on Falls in Frail Elders Living at Home in Rural Communities. Journal of Korean Academy of Nursing. 2013 Oct 1;43(5).

[6] Neira SR, Marques AP, Pérez IP, Cervantes RF, Costa JV. The effectiveness of Aquatic Therapy vs Land-based Therapy for Balance and Pain in Women with Fibromyalgia: a study protocol for a randomized controlled trial. BMC musculoskeletal disorders. 2017 Dec;18(1):22.

[7] Zhu Z, Cui L, Yin M, Yu Y, Zhou X, Wang H, Yan H. Hydrotherapy vs. conventional land-based exercise for improving walking and balance after stroke: a randomized controlled trial. Clinical rehabilitation. 2016 Jun;30(6):58793. 
[8] Pinto C, Salazar AP, Marchese RR, Stein C, Pagnussat AS. Is hydrotherapy effective to improve balance, functional mobility, motor status, and quality of life in subjects with Parkinson's disease? A systematic review and metaanalysis. PM\&R. 2018 Sep 25.

[9] Silsupadol P, Shumway-Cook A, Lugade V, van Donkelaar P, Chou LS, Mayr U, Woollacott MH. Effects of singletask versus dual-task training on balance performance in older adults: a double-blind, randomized controlled trial. Archives of physical medicine and rehabilitation. 2009 Mar 1;90(3):381-7.

[10] Masui T, Hasegawa Y, Matsuyama Y, Sakano S, Kawasaki M, Suzuki S. Gender differences in platform measures of balance in rural community-dwelling elders. Archives of gerontology and geriatrics. 2005 Sep 1;41(2):201-9.

[11] Berger L, Klein C, Commandeur M. Evaluation of the immediate and midterm effects of mobilization in hot spa water on static and dynamic balance in elderly subjects. InAnnales de réadaptation et de médecine physique 2008 Mar 1 (Vol. 51, No. 2, pp. 90-95). Elsevier Masson.

[12] Azimzadeh E, Aslankhani MA, Shojaei M, Salavati M. Effect of Hydrotherapy on Static and Dynamic Balance in Older Adults: Comparison of Perturbed and Non- Perturbed Programs. Iranian Journal of Ageing. 2013 Jan $15 ; 7(4): 27-34$.

[13] Bonnewyn A, Shah A, Bruffaerts R, Demyttenaere K. Factors determining the balance between the wish to die and the wish to live in older adults. International journal of geriatric psychiatry. 2017 Jun;32(6):685-91.

[14] Verfaillie DF, Nichols JF, Turkel E, Hovell MF. Effects of resistance, balance, and gait training on reduction of risk factors leading to falls in elders. Journal of Aging and Physical Activity. 1997 Jul;5(3):213-28.

[15] Wolfson L, Whipple R, Derby C, Judge J, King M, Amerman P, Schmidt J, Smyers. D. Balance and strength training in older adults: intervention gains and Tai Chi maintenance. Journal of the American Geriatrics Society. 1996 May;44(5):498- 506.

[16] Mudiyanselage D, Bandara PA, Croos AP. Different kind of rehabilitation exercises to reduce the number of falls among senior citizens-A literature review

[17] Anderson RL, Fishback E. Balance specific training in water and on land in older adults: a pilot study. International Journal of Aquatic Research and Education. 2010;4(3):8.

[18] Chacko TV, Thangaraj P, Muhammad GM. Epidemiology of fall and its risk factors among elders in a rural area of Coimbatore, India. International Journal Of Community Medicine And Public Health. 2017 Sep 22;4(10):3864-9.

[19] Krishnaswamy DB, Usha DG. Falls in older people national/regional review. Department of Geriatric Medicine, Madras Medical College, India. 2008.

[20] Caminiti G, Volterrani M, Marazzi G, Cerrito A, Massaro R, Sposato B, Arisi A, Rosano G. Hydrotherapy added to endurance training versus endurance training alone in elderly patients with chronic heart failure: a randomized pilot study. International journal of cardiology. 2011 Apr 14;148(2):199-203.

[21] Sherrington C, Tiedemann A. Physiotherapy in the prevention of falls in older people. Journal of physiotherapy. 2015 Apr 1;61(2):54-60.

[22] Methajarunon P, Eitivipart C, Diver CJ, Foongchomcheay A. Systematic review of published studies on aquatic exercise for balance in patients with multiple sclerosis, Parkinson's disease, and hemiplegia. Hong Kong Physiotherapy Journal. 2016 Dec 1;35:12-20.

[23] Bergamin M, Ermolao A, Tolomio S, Berton L, Sergi G, Zaccaria M. Water-versus land-based exercise in elderly subjects: effects on physical performance and body composition. Clinical interventions in aging. 2013;8:1109.

[24] Taheri MO. Effect of hydrotherapy on lower body strength and balance among elderly women. Journal of Physical Education Research. 2015;2(4):19-26.

[25] Melzer I, Elbar O, Tsedek I, Oddsson LI. A water-based training program that include perturbation exercises to improve stepping responses in older adults: study protocol for a randomized controlled cross-over trial. BMC Geriatrics. 2008 Dec;8(1):19.

[26] Lord S, Mitchell D, Williams P. Effect of water exercise on balance and related factors in older people. Australian Journal of Physiotherapy. 1993 Jan 1;39(3):217- 22.

[27] Volpe D, Giantin MG, Maestri R, Frazzitta G. comparing the effects of hydrotherapy and land-based therapy on 
balance in patients with Parkinson's disease: a randomized controlled pilot study. Clinical rehabilitation. 2014 Dec;28(12):1210-7.

[28] Alikhajeh Y, Hosseini SR, Moghaddam A. Effects of hydrotherapy in static and dynamic balance among elderly men. Procedia-Social and Behavioral Sciences. 2012 Jan 1;46:2220-4.

[29] Avelar NC, Bastone AC, Alcântara MA, Gomes WF. The effectiveness of aquatic and non-aquatic lower limb muscle endurance training in the static and dynamic balance of elderly people. Brazilian Journal of Physical Therapy. 2010 Jun;14(3):229-36.

[30] Badawy, Wanees M A and MOHAMED B. IBRAHEM. Comparing the Effects of Aquatic and Land-Based Exercises on Balance and Walking in Spastic Diplegic Cerebral Palsy Children. Medical Journal of Cairo University.2016 March; 84-1. 